\title{
Molecular Docking studies of Synthetic and Natural compounds against cFLIP protein in cancer
}

\author{
C. Nagamani ${ }^{1}$, K. Devi ${ }^{1}$, G. Sridharbabu ${ }^{2}$, Vadlakonda Rajashekar ${ }^{3}$, Sreenivas Enaganti $^{4 *}$ \\ ${ }^{1}$ Department of Pharmaceutical Chemistry, Bhaskar Pharmacy College, Hyderabad- 500034, Telangana, India \\ ${ }^{2}$ Department of Pharmaceutical Chemistry, Pragathi Pharmacy College, Pemberthy, Jangaon, Warangal-503123, Telangana, India \\ ${ }^{3}$ Department of Pharmaceutical Chemistry, Vikas College of Pharmacy, Jangaon, Warangal-503123, Telangana, India. \\ ${ }^{4}$ Department of Bioinformatics, Averinbiotech, Windsor Plaza, Nallakunta, Hyderabad-500044, Telangana, India
}

\author{
ARTICLE INFO \\ Article history: \\ Received on: 10/08/2016 \\ Accepted on: 10/12/2016 \\ Available online: 30/03/2017 \\ Key words: \\ Apoptosis, Autoimmunity, \\ Cancer, c- FLIP, Docking, \\ GOLD etc.
}

\begin{abstract}
Cellular FLICE - inhibitory proteins (c-FLIP) plays a major role in apoptosis. There are a few diseases reported which are associated with the apoptosis mechanism such as autoimmunity, AIDS and cancer. In majority of the cancers the c-FLIP over expression is seen. The aim of the present investigation is to evaluate a ligand / drug molecule which could resist the over expression of the c-FLIP and hence establishing itself as the major therapeutic drug target. In this quest, the docking of the c-FLIP with 28 ligands on GOLD software was performed. The docking results were found to be very encouraging with doxorubicin elevating itself as the ace ligand with the highest fitness score of 55.81 .
\end{abstract}

\section{INTRODUCTION}

Caspase- 8 activation is essential and plays a pivotal role in the apoptotic cell death (Wang et al., 2008). Caspases are cysteine dependent aspartate specific proteases act by splitting the substrates in a very systematic, highly specific and in a much regulated manner (Fuentes-Prior and Salvesen, 2004). It was noticed that apoptosis occurs in intrinsic and extrinsic pathways (Micheau, 2003). Activation of intrinsic pathway occurs by the formation and release of proapototic molecules which in turn activates the Apaf- 1, referring to Apoptotic Protease Activating Factor -1 (Zou et al., 1999). In contrast extrinsic pathway activates by stimulation of the cell membrane associated with death receptor (DR) (Olivier, 2003). So far 8 death receptors have been identified amongst which TRAIL- R1/R2 and Fas

\section{* Corresponding Author}

Sreenivas Enaganti, Department of Bioinformatics, Averinbiotech, Windsor Plaza, Nallakunta, Hyderabad-500044, Telangana, India. Email: sreenivas.bioinfo@gmail.com
(Inazawa et al., 1992 ; Oehm et al., 1992; Macfarlane et al.,1997; Screaton et al., 1997; Sheridan et al., 1997; Wu et al., 1997), when are bound to their cognate ligands, activates the adaptor molecule FADD (MORT1) (Chinnaiyan et al., 1996) forms the Death- Inducing Signalling Complex (DISC) during which the caspase- 8 is activated (Kischkel et al., 1995). Initiator caspase -8 is composed of FLICE, MACH, and MCH5 (Muzio et al., 1996).

C-FLIP are also known by different names like FLAME1, I - FLICE, CLARP, CASH, MRIT, usurpin and Casper (Goltsev et al.,1997; Inohara et al.,1997; Rasper et al., 1998; Han et al.,1997; Hu et al., 1997) are present on2q33-34 chromosome along with caspase- 8 and caspase-10 (Rasper et al., 1998). On the

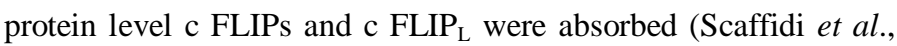
1999). Structurally it was noted that the c FLIP lacks the amino acid residue which are crucial for caspase activity (Andreas et al., 2001). Known as a major anti-apoptotic regulator, c FLICE works by inhibiting and regulating the cell death by the death receptor Fas, DR 4, DR 5 and TNFR1 (Sarah et al., 2013). 
There are reports of c FLIP over- expression associated with several cancers like colon rectal cancer, cervical cancer, Burkitt's lymphoma and non-Hodgkinis lymphoma (Djerbi et al., 1999; Wang et al., 2007; Ullenhag et al., 2007; Valnet-Rabier et al., 2005). C FLIP that inhibits TRAIL induced cell death is seen as a tumor- progression factor. Not only in cancers, c FLIP also has an impressive role to be portrayed in cardiovascular diseases (Valente et al., 2006), multiple sclerosis (Antel, 1999), leading to myelin damage, Rheumatoid arthritis (Janossy et al., 1981), Alzheimer's diseases (De Felice and Ferreira, 2002) and diabetes mellitus (Hilliard et al., 2001). These observations emphasize on c FLIP being a potential drug target for cancers as many types of cancers associated with the over expression of c FLIP. The aim of the present article is to identify a drug molecule which could resist the over expression of the $\mathrm{c}$ FLIP and hence regulating the cancers.

\section{MATERIALS AND METHODS}

\section{Protein selection and preparation}

The crystal structure of human c- FLIP protein (PDB ID: 3 H13) was retrieved from protein data bank with a resolution of $2.2 \AA$ A. Preparation of the retrieved protein was performed by using prepare protein protocol of Discovery Studio (DS). Initially all the water molecules were removed and hydrogens were added to satisfy the valances. Energy minimization of the protein structure was done by employing CHARMm forcefields and smart minimization algorithm followed by conjugant gradient algorithm in DS until the convergence gradient is satisfied.

\section{Ligand selection and preparation}

28 ligands were selected from various literature sources ( Safa and Pollok, 2011; Noh et al., 2012 ; McCourt et al., 2012 ; Zhang et al., 2008 ; Yodkeeree et al., 2009). Using Chemsketch (ACDLABS 12.0), the selected compounds are drawn and saved in mol file format. The saved ligand compounds were later imported in DS and further ligand preparation with constraint parameters such as ionization change, tautomer and isomer generation and all the duplicate structures are removed followed by geometry optimization and energy minimization of the lowest energy structure using the CHARMm forcefield. The individual compounds were finally converted to $3 \mathrm{D}$ structures using DS catalyst algorithm.

\section{Docking}

Genetic Optimization Ligand Docking-GOLD, one of the finest molecular docking mechanisms, used to dock the selected ligands into the active site of the protein c-FLIP. GOLD uses Genetic Algorithm which renders ligand full flexibility and partial flexibility to proteins. This algorithm is an optimizing method, allowing the direct random searches (Shahper and Asad, 2008) utilizing the electronic, lipophilicity and shaped related properties. To simplify the docking calculations the protein's active site is defined within $10 \AA$ radius and the amino acids present within this radius are used as an input to run GOLD docking calculations with default parameters. The default evolutionary parameters used are population size: 1/4100; selection pressure: 1/4 1.1; operations: $1 / 4$ 100,000; islands: $1 / 4$ 5; niche size: $1 / 42$; migration: $1 / 410$; mutation: $1 / 495$; crossover: $1 / 495$. Using the default GOLD fitness function, ten docking runs were performed per ligand compound unless three of the 10 poses were within $1.5 \AA$ RMSD of each other with an early termination of Generic Optimization calculation is enabled. The ability of ligand compound interaction with the protein ability of a compound depends on the fitness score, greater the GOLD fitness score better the binding affinity. Ligand compounds which showed the expected interactions with the amino acids present in the active site of the protein, and with high binding scores were selected as potent inhibitors of c-FLIP. The best pose of each ligand was chosen based on the GOLD fitness score and visualized using silver.

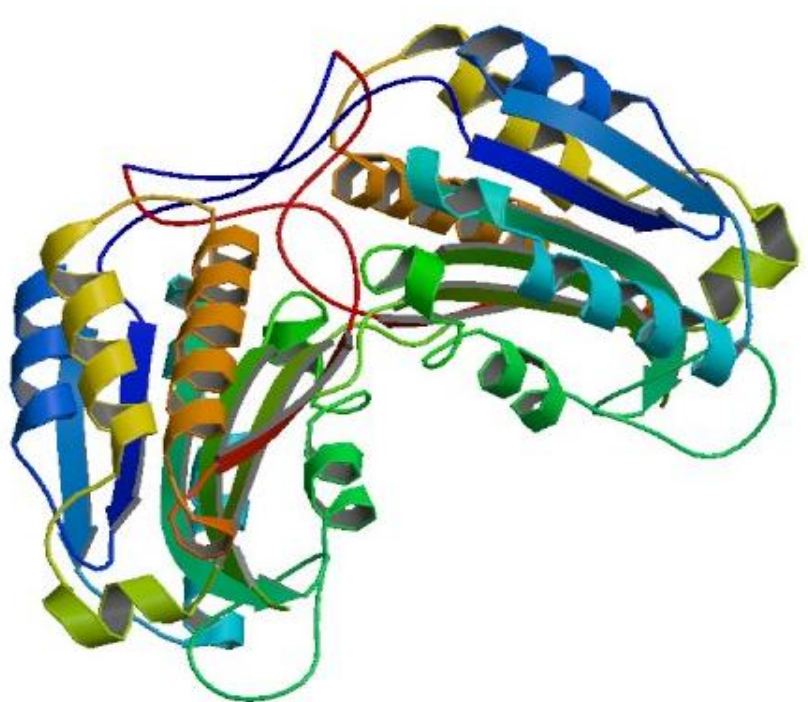

Fig. 1: Secondary Structure of human c- FLIP (PDB ID: 3H13).

\section{RESULTS AND DISCUSSION}

Docking studies was performed using GOLD to find the interactions between the active site of c-FLIP and ligands. The prepared protein and the ligands were imported on to the GOLD software facilitating them to undergo flexible docking process commenced with default parameters. For each ligand, 10 different poses with their corresponding GOLD fitness scores were saved. The selection of best ligand pose was called on their affinity towards the amino acids which renders them the highest fitness score. Higher the GOLD fitness score of the ligand pose was better because it was calculated based on the negative of the sum of the component energy terms. The optimized fitness function was used for the prediction of well fitted ligand binding position that has the least energy with average Gold fitness score. From this analyzes it was elucidated that the Doxorubicin and Sorafenib as the active molecules against c-FLIP which shows the good fitness score and is selected as potent inhibitors which are further validated by using the visualization method to find the suitable binding mode of the inhibitor based on the critical interactions with the active site 
residues. Silver was used to visualize the protein ligand complex interactions. From the table 1, it is therefore noted that the ligand Doxorubicin and Sorafenib has the highest fitness scores of 55.81 and 52.28. From the figure 2, it can be elucidated that the interacting amino acids are Val 324, Asp 325, Arg 315 and Trp 322. The ligand pose was further analyzed and studied based on their hydrogen bond interaction as the hydrogen bonding plays a pivotal role in the proteins structural and biological functions. Close observation of the interactions revealed that the Try 322 forms a Hydrogen bond with the $\mathrm{OH}$, Asp 325 with O, Val 324 with $\mathrm{O}$ and Arg 315 forms the hydrogen bond with NH1 respectively.

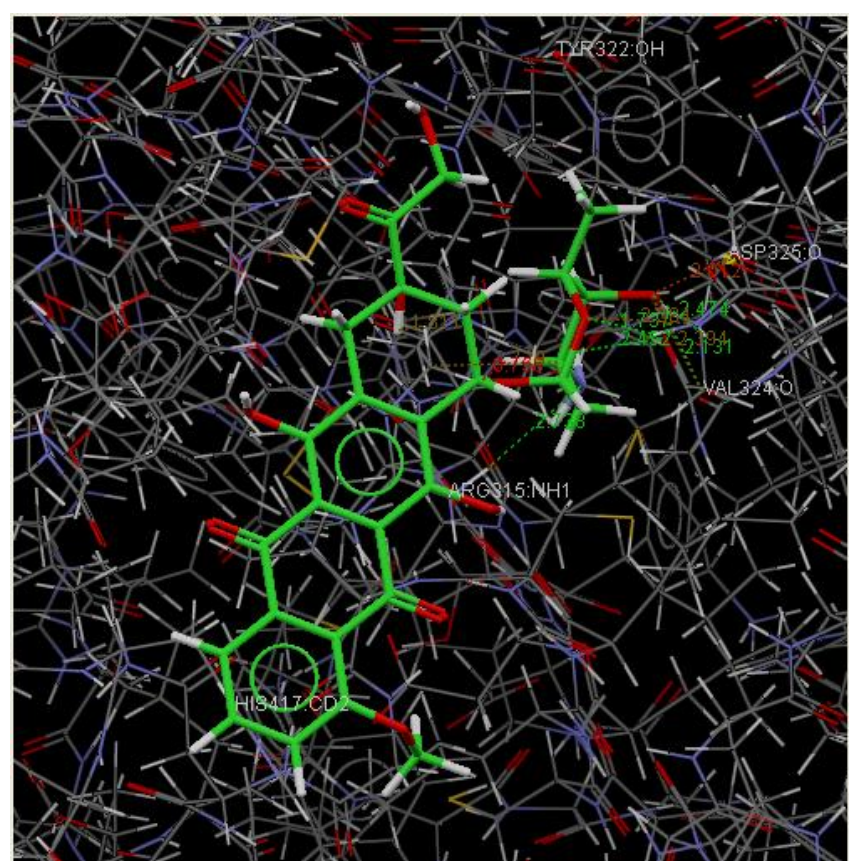

Fig. 2: Shows H-bonding interactions of Doxorubicin with active site residues of human c- FLIP (PDB ID: 3H13).

Table 1: Protein-Ligand fitness scores

Ligand name $\quad$ Fitness $\quad$ S(hb_ext) $\quad$ S(vdw_ext) $\quad$ S(hb_int) $\quad$ S(int)

\begin{tabular}{lccccc}
\hline 5-Fluorouracil & 15.96 & 0.25 & 11.43 & 0.00 & 0.00 \\
9-NC & 32.55 & 0.00 & 26.91 & 0.00 & -4.45 \\
Camptothecin & 38.18 & 0.81 & 28.14 & 0.00 & -6.63 \\
Celastrol & 34.75 & 6.00 & 25.29 & 0.00 & -30.12 \\
Celecoxib & 43.55 & 6.00 & 28.88 & 0.00 & -10.81 \\
Chrysin & 31.47 & 1.32 & 22.97 & 0.00 & -7.13 \\
Anisomycin & 41.79 & 6.00 & 29.10 & 0.00 & -21.10 \\
Cisplatin & -501.36 & 0.00 & 79.83 & 0.00 & -3055.64 \\
Cycloheximide & 36.96 & 6.00 & 23.99 & 0.00 & -10.11 \\
Etoposide & 46.17 & 6.00 & 34.00 & 0.00 & -32.87 \\
Flavopiridol & 43.63 & 7.75 & 29.22 & 0.00 & -21.50 \\
Nocodazole & 40.21 & 6.00 & 26.15 & 0.00 & -8.72 \\
Paclitaxel & -1196.97 & 5.29 & 76.69 & 0.00 & -6538.54 \\
Sorafenib & 52.28 & 7.82 & 34.84 & 0.00 & -17.25 \\
Doxorubicin & 55.81 & 1.73 & 43.69 & 0.00 & -29.96 \\
Droxinostat & 40.10 & 6.83 & 26.04 & 0.00 & -12.73 \\
Genistein & 33.48 & 2.00 & 24.23 & 0.00 & -9.22 \\
Irinotecan & 47.38 & 0.00 & 38.29 & 0.00 & -26.31 \\
\hline
\end{tabular}

\begin{tabular}{lccccc}
\hline Lupeol & 21.92 & 0.00 & 22.74 & 0.00 & -46.75 \\
Oxaliplatin & -18.69 & 6.00 & 29.36 & 0.00 & -325.28 \\
Quinacrine & 27.58 & 2038 & 38.73 & 0.00 & -140.31 \\
Silybin & 44.70 & 7.32 & 31.27 & 0.00 & -28.04 \\
Taxol & -1203.03 & 5.86 & 67.57 & 0.00 & -6508.96 \\
Trichostatin A & 43.76 & 5.72 & 31.85 & 0.00 & -28.83 \\
valproic acid & 29.43 & 6.00 & 19.94 & 0.00 & -19.95 \\
Vorinostat & 46.66 & 6.00 & 32.64 & 0.00 & -21.13 \\
Withaferin & 35.15 & 0.00 & 29.67 & 0.00 & -28.21 \\
Zerumbone & 26.52 & 0.00 & 19.80 & 0.00 & -3.49 \\
\hline
\end{tabular}

Figure 3 shows the protein docking to the ligand Sorafenib. The interacting amino acids are Val 324, Trp 322 and His 417 and the Hydrogen bonds were observed between Val 324 and $\mathrm{O}$, Trp 322 binding with $\mathrm{OH}$ and subsequently, His 417 binding to $\mathrm{C} / \mathrm{OD} 2$.

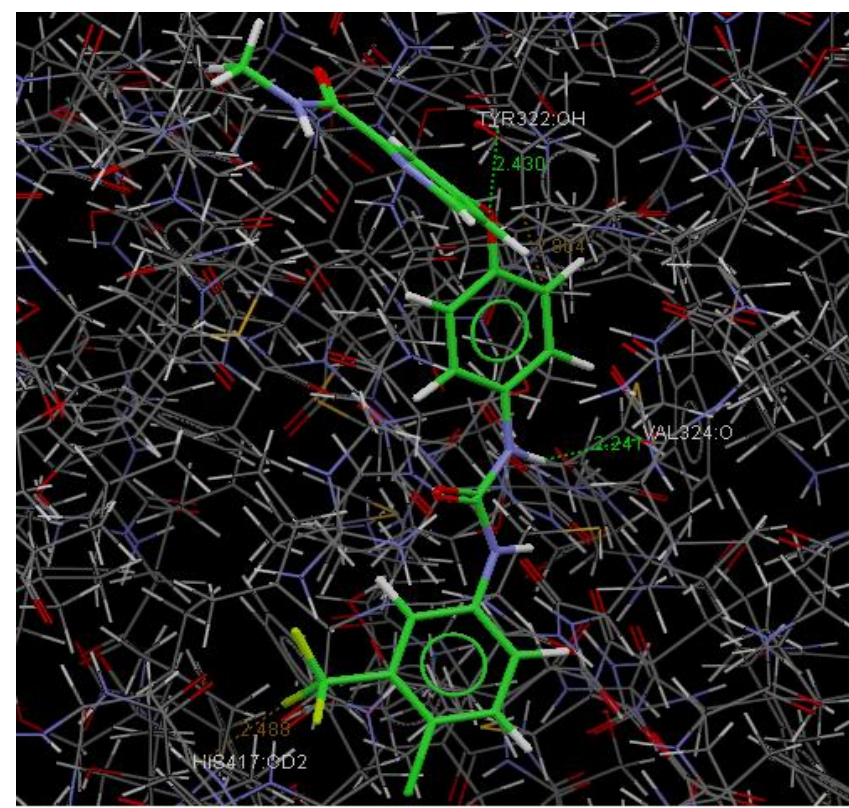

Fig. 3: Shows H-bonding interactions of Sorafenib with active site residues of human c- FLIP (PDB ID: 3H13).

\section{CONCLUSION}

Cancer is one of the major causes of death in the present day. Researchers are involved in finding a solution for this disease. It is known from the reports that the c FLIP is a cellular protein whose levels are elevated during certain types of cancers. Not only in cancers, it's over expression has also been noticed in other health associated diseases as reported in the article making c-FLIP a prime drug target. Hence, therefore, we tried to evaluate a ligand molecule which could inhibit its over expression by targeting the c-FLIP with 28 ligands. The GOLD fitness scores were very promising with the highest fitness score of 55.81 and 52.28 for Doxorubicin and Sorafenib respectively. Henceforth, monitoring the changes in the levels of c-FLIP could be of great diagnostic value.

Financial support and sponsorship: Nil.

Conflict of Interests: There are no conflicts of interest. 


\section{REFERENCES}

Andreas Krueger, Sven Baumann, Peter H. Krammer, Sabine Kirchhoff: FLICE-Inhibitory Proteins: Regulators of Death Receptor-Mediated Apoptosis. Mol. Cell. Biol, 2001; 21(24):8247.

Antel J. Multiple sclerosis--emerging concepts of disease pathogenesis. J Neuroimmunol, $1999 ; 98: 45-48$

Chinnaiyan AM, Tepper CG, Seldin MF. FADD/MORT1 is a common mediator of CD95 (Fas/APO-1) and tumor necrosis factor receptorinduced apoptosis . J Biol Chem, $1996 ; 271: 4961$ - 4965

De Felice FG, Ferreira ST. Beta-amyloid production, aggregation, and clearance as targets for therapy in Alzheimer's disease . Cell Mol Neurobiol . $2002 ; 22: 545-563$

Djerbi M, Screpanti V, Catrina AI, Bogen B, Biberfeld P, Grandien A. The inhibitor of death receptor signaling, FLICE-inhibitory protein defines a new class of tumor progression factors. The Journal of experimental medicine, $1999 ; 190(7): 1025-32$.

Fuentes-Prior P, Salvesen GS. The protein structures that shape caspase activity, specificity, activation and inhibition. Biochemical Journal, 2004; 384(2):201-32.

Goltsev YV, Kovalenko AV, Arnold E, Varfolomeev EE, Brodianskii VM, Wallach D. CASH, a novel caspase homologue with death effector domains. Journal of Biological Chemistry, 1997; 272(32):19641-4.

Han DK, Chaudhary PM, Wright ME, Friedman C, Trask BJ, Riedel RT, Baskin DG, Schwartz SM, Hood L. MRIT, a novel death-effector domain-containing protein, interacts with caspases and BclXL and initiates cell death. Proceedings of the National Academy of Sciences, 1997; 94(21):113338 .

Hilliard B, Wilmen A, Seidel C. Roles of TNF-related apoptosisinducing ligand in experimental autoimmune encephalomyelitis . J Immunol, $2001 ; 166: 1314-1319$

Hu S, Vincenz C, Ni J, Gentz R, Dixit VM. I-FLICE, a novel inhibitor of tumor necrosis factor receptor-1-and CD-95-induced apoptosis. J. Biol. Chem, 272:17255-17257.

Huang SY, Zou X. Advances and challenges in protein-ligand docking. International journal of molecular sciences, 2010;11(8):3016-34.

Inazawa J, Itoh N, Abe T, Nagata S. Assignment of the human Fas antigen gene (Fas) to 10q24. 1. Genomics, 1992;14(3):821-2.

Inohara N, Koseki T, Hu Y, Chen S, Núñez G. CLARP, a death effector domain-containing protein interacts with caspase- 8 and regulates apoptosis. Proceedings of the National Academy of Sciences, 1997 ;94(20):10717-22.

Janossy G, Panayi G, Duke O. Rheumatoid arthritis: a disease of Tlymphocyte/macrophage immunoregulation . Lancet, $1981 ; 2: 839-842$

Kischkel FC, Hellbardt S, Behrmann I, Germer M, Pawlita M, Krammer PH, Peter ME. Cytotoxicity-dependent APO-1 (Fas/CD95)associated proteins form a death-inducing signaling complex (DISC) with the receptor. The EMBO journal, 1995; 14(22):5579.

Macfarlane M , Ahmad M, Srinivasulu SM. Identification and molecular cloning of two novel receptors for the cytotoxic ligand TRAIL . J Biol Chem, $1997 ; 272: 25417$ - 25420.

Manoj Kumar Annamala, Krishna Kishore Inampudi and Lalitha Guruprasad Docking of phosphonate and trehalose analog inhibitors into $M$. tuberculosis mycolyltransferase $\mathrm{Ag} 85 \mathrm{C}$ : Comparison of the two scoring fitness function GoldScore and ChemScore, in the GOLD software: Bioinformation, 2007; 1(9): 339-350.

McCourt C, Maxwell P, Mazzucchelli R, Montironi R, Scarpelli M, Salto-Tellez M, O'Sullivan JM, Longley DB, Waugh DJ. Elevation of c-FLIP in castrate-resistant prostate cancer antagonizes therapeutic response to androgen receptor-targeted therapy. Clinical Cancer Research, 2012 ;18(14):3822-33.

Micheau O. Cellular FLICE-inhibitory protein: an attractive therapeutic target?. Expert opinion on therapeutic targets, 2003;7(4):559-73.

Muzio M, Chinnaiyan AM, Kischkel FC, O'Rourke K, Shevchenko A, Ni J, Scaffidi C, Bretz JD, Zhang M, Gentz R, Mann M. FLICE, a novel FADD-homologous ICE/CED-3-like protease, is recruited to the CD95 (Fas/APO-1) death-inducing signaling complex. Cell, 1996; 85(6):817-27.

Noh HJ, Lee SJ, Sung EG, Song IH, Kim JY, Woo CH, Kwon TK, Lee TJ. CHOP down-regulates cFLIPL expression by promoting ubiquitin/proteasome-mediated cFLIPL degradation. Journal of cellular biochemistry, $2012 ; 113(12): 3692-700$.

Oehm A, Behrmann I, Falk W . Purification and molecular cloning of the APO-1 cell surface antigen, a member of the tumor necrosis factor/nerve growth factor receptor superfamily. Sequence identity with the Fas antigen . J Biol Chem , $1992 ; 267: 10709$ - 10715

Rasper DM, Vaillancourt JP, Hadano S, Houtzager VM, Seiden I, Keen SL, Tawa P, Xanthoudakis S, Nasir J, Martindale D, Koop BF, Peterson EP, Thornberry NA, Huang J, MacPherson DP, Black SC, Hornung F, Lenardo MJ, Hayden MR, Roy S and Nicholson DW. Cell death attenuation by'Usurpin', a mammalian DED-caspase homologue that precludes caspase-8 recruitment and activation by the CD-95 (Fas, APO-1) receptor complex. Cell Death Differ, 1998; 5:271-288.

Safa AR, Pollok KE. Targeting the anti-apoptotic protein c-FLIP for cancer therapy. Cancers, 2011;3(2):1639-71

Sarah Shirley, Olivier Micheau: Targeting c-FLIP in cancer. Cancer Letters 2013; 332(2): 141-50

Scaffidi C, Schmitz I, Krammer PH, Peter ME. The role of c-FLIP in modulation of CD95-induced apoptosis. J Biol Chem , 1999;274(3):1541-8.

Screaton GR , Mongkolsapaya J, Xu Xn . TRICK2, a new alternatively spliced receptor that transduces the cytotoxic signal from TRAIL . Curr Biol , 1997 ; 7: 693 - 696.

Shahper NK and Asad UK. Computational simulation of mitoxantrone binding with human serum albumin. J. Proteomics Bioinforma, 2008; 1 : 17-20.

Sheridan JP , Marsters SA, Pitti RM . Control of TRAIL-induced apoptosis by a family of signaling and decoy receptors . Science, $1997 ; 277$ : $818-821$

Ullenhag GJ, Mukherjee A, Watson NF, Al-Attar AH, Scholefield JH, Durrant LG. Overexpression of FLIPL is an independent marker of poor prognosis in colorectal cancer patients. Clinical Cancer Research, 2007;13(17):5070-5.

Valente G, Manfroi F, Peracchio C, Nicotra G, Castino R, Nicosia G, Kerim S, Isidoro C. cFLIP expression correlates with tumour progression and patient outcome in non-Hodgkin lymphomas of low grade of malignancy. British journal of haematology., 2006;132(5):560-70.

Valnet-Rabier MB, Challier B, Thiebault S, Angonin R, Margueritte G, Mougin C, Kantelip B, Deconinck E, Cahn JY, Fest T. c-Flip protein expression in Burkitt's lymphomas is associated with a poor clinical outcome. British journal of haematology, 2005 ;128(6):767-73.

Wang F, Du L, Wang X: TNF-alpha induces two distinct caspase-8 activation pathways. Cell, 2008; 133:693-703.

Wang W, Wang S, Song X, Sima N, Xu X, Luo A, Chen G, Deng D, Xu Q, Meng L, Lu Y. The relationship between c-FLIP expression and human papillomavirus E2 gene disruption in cervical carcinogenesis Gynecologic oncology, 2007;105(3):571-7.

Wu Gs, Burns TF, Mc Donald ER 3 RD. KILLER/DR5 is a DNA damage-inducible p53-regulated death receptor gene . Nat Genet, 1997 ; 17 $141-143$

Yodkeeree S, Sung B, Limtrakul P, Aggarwal BB. Zerumbone Enhances TRAIL-Induced Apoptosis through the Induction of Death Receptors in Human Colon Cancer Cells: Evidence for an Essential Role of Reactive Oxygen Species. Cancer research, 2009;69(16):6581-6589.

Zhang G, Park MA, Mitchell C, Hamed H, Rahmani M, Martin AP, Curiel DT, Yacoub A, Graf M, Lee R, Roberts JD. Vorinostat and sorafenib synergistically kill tumor cells via FLIP suppression and CD95 activation. Clinical cancer research, 2008 ;14(17):5385-99.

Zou $\mathrm{H}$, Li Y,Liu X, Wang X . An APAF-1. cytochrome c multimeric complex is a functional apoptosome that activates procaspase-9. J Biol Chem , $1999 ; 274: 11549$ - 11556

\section{How to cite this article:}

Nagamani C, Devi K, Sridharbabu G, Rajashekar V, Enaganti S. Molecular Docking studies of Synthetic and Natural compounds against cFLIP protein in cancer. J App Pharm Sci, 2017; 7 (03): 109-112. 